\title{
Evolución de las Tendencias en la Gestión de Recursos Humanos
}

\author{
Alicia Cristina Silva Calpa ${ }^{1}$ \\ Jorge Briceño López ${ }^{2}$ \\ Martha de Jesús Guerrero Guzmán ${ }^{3}$
}

\section{Resumen}

La gestión de recursos humanos y su directa relación con los resultados obtenidos por las organizaciones, es uno de los temas más estudiados en los últimos años y que han adquirido importancia, cuando se empezó a reconocer el papel estratégico que desempeña el capital humano en la empresa. Hechos como la globalización, el desarrollo de las comunicaciones y la internacionalización de procesos productivos han permitido que las empresas se esfuercen por la búsqueda de la competitividad para mantenerse activos en los mercados, lo que ha propiciado la evolución en la gestión de recursos humanos y todas las tendencias que lo orientan.

Cuando se habla de la complejidad laboral se hace necesario tomar en cuenta los factores personales y ambientales que puedan incidir en las actuaciones del trabajador. Para la dirección estos factores son entendidos como el proceso de influir en la gente para que contribuya a alcanzar los objetivos de las personas y de la organización en conjunto y requiere la creación y el mantenimiento de un contexto en el que los individuos trabajen juntos, hacia el logro de objetivos comunes.

\footnotetext{
${ }^{1}$ Administradora de empresas, Especialista en Pedagogía. Mg. Sistemas de Calidad y Productividad. Universidad Nacional Abierta y Distancia UNAD. alicia.silva@unad.edu.co.

${ }^{2}$ Economista, Especialista, Mg. En Administración de Empresas. Universidad Nacional Abierta y Distancia UNAD. jorge.briceno@unad.edu.co.

${ }^{3}$ Administradora de empresas, Especialista en Pedagogía. Mg. en Administración de Empresas. Universidad Nacional Abierta y Distancia UNAD. martha.guerrero@unad.edu.co.
} 


\section{Introducción}

Para el manejo del recurso humano se han propuesto nuevos estilos de liderazgo y prácticas administrativas de alta eficiencia y desempeño, orientados al mejoramiento continuo; haciendo que el proceso de gestión de personal haya evolucionado en todas sus facetas, tanto para el reclutamiento, con nuevas formas de identificar talentos; en la contratación, con la flexibilidad del empleo, las formas de empleo temporal, los empleos parciales, el outsourcing; en el proceso de selección, con las evaluaciones y test de personalidad, de evaluación por competencias; en el proceso de capacitación, identificando nuevas formas de cognición, aprendizaje colaborativo, aplicación de las tecnologías de la información y la comunicación, la adquisición de nuevas habilidades; en el desarrollo de personal, con la identificación y retención de talentos de alto potencial, internacionalización de las prácticas de recursos humanos, el uso de la computación en la nube y las nuevas tendencias teóricas aplicadas, para enfrentar los desafíos de los mercados actuales.

\section{Tendencias de la gestión de recursos humanos}

Después de todos los avances teóricos y prácticos en recursos humanos, la ciencia de gestionar personas, continúa en evolución ya que las personas propician que las empresas tengan su propia impronta, compuesta por los comportamientos individuales que le permiten ser un ente que evoluciona y crece con las innovaciones de sus empleados, que, en condiciones óptimas, responden de forma creativa con nuevas formas de hacer las cosas, para las nuevas tendencias del entorno organizacional. Es así como los administradores de personal, deben afrontar nuevos desafíos orientados a las nuevas tendencias de la comunicación, la incorporación de las tecnologías y de almacenamiento de información, la tendencias de investigación para la innovación, las técnicas de compensación, 
reconocimiento de potenciales y retención mediante la motivación; el acierto en la toma de decisiones, la flexibilidad laboral y de contratación, contando con ventaja comparativa en su capital humano, enriquecido de forma constante, mediante el aprendizaje.

\section{El aprendizaje organizacional.}

Se considera el aprendizaje organizacional como un proceso mediante el cual las organizaciones transforman información en conocimiento, lo difunden y explotan con el fin de incrementar su capacidad innovadora y competitiva y es importante para el incremento del capital intelectual de la empresa, para la solución de dificultades con el fin de adaptarse al entorno cambiante y competitivo. Ahumada F.L. (2002) expresa que es la habilidad para cambiar la lógica dominante de la organización y, por tanto, la capacidad de aprendizaje organizacional entendida como un proceso emergente sería la clave del éxito de la supervivencia organizacional dado la dificultad de saber con anticipación la dirección que tomarán los cambios del entorno organizacional. En cuanto a las organizaciones se manifiesta que compiten por recursos escasos en un entorno que selecciona a aquellas que cuentan con más y mejores capacidades para hacer frente a sus demandas, eliminando a las menos competentes en esta perspectiva donde el conocimiento y el aprendizaje organizacional aparecen como la principal fuente de competitividad de la empresa y aparece como una herramienta capaz de generar dichas ventajas competitivas, ya que en él radica la identidad misma de la organización.

\section{Gestión de Recursos Humanos}

Los procesos desarrollados dentro de la administración de recursos humanos, gestión del talento humano o gestión humana, como se denomina actualmente, han tomado gran importancia dentro de las organizaciones y para García (2009) la han orientado a 
desarrollarse y definirse como un conjunto de procesos estratégicos, ya que se afirma que el ser humano es el factor que da ventaja competitiva a la organización. Sobre los procesos que componen el desarrollo de la gestión del talento humano, García (2009) los identifica como la planificación del talento humano, el análisis y diseño de puestos de trabajo, la cobertura de las necesidades del talento humano de la organización, el reclutamiento y la selección, la iniciación de los nuevos empleados en la organización, la socialización, la capacitación para empleados nuevos en un puesto, la mejora del desempeño en el trabajo, la obtención de cooperación creativa y el desarrollo de buenas relaciones de trabajo, el aumento del potencial y desarrollo del individuo, la evaluación de la actuación de los empleados, la retribución de los empleados a través de incentivos y beneficios, la gestión de la salud e higiene en el trabajo, el monitoreo a través de sistemas, entre otras.

\section{Recursos Humanos y Gestión del Conocimiento.}

Con frecuencia se hace necesario prestar mayor atención a los activos inmateriales de la organización, sobre todo de aquellos capaces de aportar valor económico a la empresa y el conocimiento se ha convertido en uno de los activos más importantes para las organizaciones, en la búsqueda de alcanzar ventajas competitivas en el mercado. Sobre este hecho Schwartzman K.R. (2000) expresa, que en un modelo flexible y dentro de una estrategia de mejora continua, el trabajador debe tener la capacidad de incorporar y aportar sus conocimientos al proceso de producción y participar en el análisis y solución de problemas que obstaculizan la calidad y la productividad y el conocimiento del que disponen las personas en la organización y su recopilación no sólo genera valor económico para la empresa, sino que, en la nueva era de la información, es el activo para marcar la diferencia. 
El conocimiento se considera un nuevo capital y su gestión hace parte de la cultura de la organización y como activo permanente es un instrumento básico para la gestión empresarial. Se busca primordialmente identificar, clasificar y usar de la forma más eficiente el conocimiento para obtener ventajas competitivas y a nivel interno de la empresa, compartir ese conocimiento aumenta los niveles de rentabilidad además que posibilita afrontar desde los problemas más simples hasta los más complejos.

\section{Flexibilidad Laboral y de Contratación}

Con la transformación de los procesos productivos y dentro de las nuevas formas de organización empresarial, las organizaciones se han adaptado a las características del mercado y han adoptado flexibilidad laboral, vista sobre todo en las nuevas formas de contratación, entre las cuales se destacan los empleos ocasionales, las agencias de trabajo temporal (ETT), las empresas de outsourcing y las opciones de empleos parciales. Las agencias de empleo temporal han tomado auge debido a diversas razones, como son, por necesidades específicas y especializadas, por el gran tamaño de la organización, por la necesidad de delegar el pago de sueldos y prestaciones entre otras. La contratación por medio de outsourcing, es preferida por las empresas que deciden dedicarse a su objeto social y dejar en manos de otra empresa, todo el proceso de gestión de personal. Estas y otras prácticas tienen sus ventajas y sus desventajas y por lo general afectan el sentido de pertencia y el empoderamiento de los empleados, pero en general traen ventajas financieras a corto y largo plazo a las empresas.

Con el fin de alcanzar ventajas en los mercados cada vez más competitivos las organizaciones han buscado alcanzar mayor productividad, aplicando modelos de flexibilidad, pues modelos que han contribuido a modificar las relaciones laborales en el 
ámbito de las organizaciones productivas; sobre este aspecto Arenas (2002) argumenta, que se han planteado tres formas principales de flexibilización: la primera es la que se refiere al monopolio en el reclutamiento de trabajadores. La segunda alude a la flexibilidad en las formas de terminación del vínculo laboral y la disminución de la estabilidad laboral. Y la tercera se relaciona con las modalidades específicas en que se presta el trabajo, las que dieron lugar a las llamadas «relaciones de trabajo atípicas»: trabajo a tiempo parcial, trabajo intermitente, contratos de duración determinada, programas de empleo formación, «teletrabajo», trabajo mediante vínculo autónomo, etc. En este grupo de aspectos de la flexibilidad en la contratación se sitúan precisamente las relaciones de trabajo triangular, y entre ellas la intermediación de las agencias de trabajo temporal.

Las empresas de trabajo temporal representan una de las máximas expresiones de flexibilización de las formas de empleo y de la propia estructura organizativa de la empresa. Dentro del modelo de descentralización productiva la utilización de ese tipo de empresas mejora la operatividad del mercado de trabajo y facilita el empleo de determinados grupos de trabajadores. Para Rodríguez, T.E. (2010) además de lo expresado permite cumplir funciones que no realiza el personal de planta por no formar parte de las actividades permanentes de la administración; desarrollar programas o proyectos de duración determinada; suplir necesidades de personal por sobrecarga de trabajo, determinada por hechos excepcionales; desarrollar labores de asesoría y consultoría institucional de duración total no superior a doce (12) meses y que guarde relación directa con el objeto y la naturaleza de la institución.

Para Díaz (2005) las empresas de trabajo temporal fueron creadas con el propósito de proveer personal temporal, ofrecer rapidez en la contratación por obra, y satisfacer 
eventos extraordinarios como picos de trabajo, por excesos de pedidos o actividades acumuladas; y para sustituir trabajadores en suspensión de su relación de trabajo.

Adicionalmente, asumen los costos indirectos de contratación, desde el reclutamiento hasta el retiro del trabajador, además que se reducen costos de personal.

Fernández(2004) argumenta que la presencia de trabajadores temporales podría provocar una reducción en los costes de búsqueda, selección y formación de trabajadores y el hecho de que una empresa decida o no utilizar trabajadores temporales, así como la proporción de ellos que tenga cada una, pueden estar condicionados por diversos factores relativos a la corporación y a su entorno, tales como su tamaño, el nivel de competencia dentro del mercado en el que opera o la incertidumbre a que se enfrenta. También pueden ser utilizadas en aquellas empresas cuya actividad productiva esté sometida a fuertes variaciones de carácter estacional, cíclico o inesperado sean más proclives a utilizar algún trabajador temporal, y que la proporción de éstos sea mayor.

En cuanto al número de trabajos temporales para Fernández(2004) cuantos más trabajadores tenga una empresa, mayor será la probabilidad de que utilice algún trabajador temporal, aunque sólo sea para cubrir vacantes transitorias de trabajadores indefinidos; por el contrario, es posible que cuanto mayor sea el tamaño de la empresa, más capacidad tenga ésta para cubrir las variaciones temporales en la carga de trabajo a través del resto de trabajadores permanentes, en lugar de tener que recurrir necesariamente a la contratación de temporales

\section{Comunicaciones y Recursos Humanos}

El uso de las comunicaciones es fundamental para la gestión de recursos humanos, para Trigueros (2014) la utilización de las TI tiene una relación positiva y estadísticamente 
significativa tanto con el concepto global de satisfacción de los recursos humanos, como con los ítems individuales que integran dicho concepto. Lo que permite confirmar que cuanto mayor es la utilización de las TI, mejores son los resultados en la satisfacción de los recursos humanos de la organización. Estas TI se utilizan en cada una de las áreas consideradas por el cuadro de mando integral como críticas para la excelencia y que hacen referencia con valoración de los clientes, satisfacción de los recursos humanos, procesos internos y resultados financieros

Sobre la eficacia de su utilización Trigueros (2014) comenta que las TI son herramientas eficaces para mejorar la percepción de los clientes sobre la los productos y servicios ofertados por la empresa, así como sobre los servicios de atención al cliente. En cuanto al segundo de los factores, los recursos humanos, la utilización de las TI reduce el estrés de los trabajadores, su absentismo y mejoran su entusiasmo e implicación, lo que permite una mayor satisfacción de los recursos humanos de la organización. En relación a los procesos internos, la utilización de las TI ejerce un efecto positivo mejorando plazos de entrega, reduciendo defectos, en definitiva, mejorando su eficacia. Hoy en día quien tiene la información tiene el poder para la toma de decisiones acertada y oportuna, por ello la gestión de recursos humanos ha aprovechado tanto el desarrollo a nivel de software como de almacenamiento de información, entre los de mayor aplicación se encuentra la computación en la nube por la capacidad de almacenamiento de grandes contenidos de datos.

Existe entonces una relación directa entre los mercados y su manejo de información, Prieto y Martínez (2004) argumentan que la permanencia en los mercados de las organizaciones, dependerá en gran medida en la forma como maneje la información basada 
en las innovaciones tecnológicas y el mejoramiento de los recursos humanos. Para alcanzar una estrategia de negocio estos constituyen los medios principales implementadas a través de la planificación estratégica, lo que conllevara a incrementos de la productividad, así se harán más competitivas y se prepararan para enfrentar los retos del futuro.

Prieto y Martínez (2004) concluyen que los sistemas de información aportan a la gerencia la transparencia necesaria para ver con detenimiento una realidad compleja: el hombre, la empresa y el entorno; permitiendo el aprovechamiento racional del talento y la profesionalidad, dar a los recursos humanos de la empresa un espacio creativo para sumar sus logros a una empresa mejor, conocer la situación de la empresa dentro de un contexto de mercado que le permita aumentar las ventajas competitivas.

\section{Sistemas de compensación en búsqueda de resultados}

En el ámbito empresarial de hoy en día, las recompensas y los reconocimientos han llegado a ser más importante que nunca ya que los gerentes disponen de menos manera de influir en sus empleados o de moldear su comportamiento. En tiempo de estrechez económica, las recompensas y el reconocimiento proporcionan una manera eficaz de estimular a los empleados para que logren más altos niveles de desempeño. Las decisiones para hacer que el rendimiento organizacional sea una condición real, deben ser planeación de forma articula a la estrategia de negocio, ya que una medida que puede buscar reducción de costos, por otro lado, puede propiciar los recortes de presupuestos en estrategias de recursos humanos. El presupuesto para los mecanismos de recompensa siempre son una inversión para la obtención de resultados, productividad, competitividad y rentabilidad.

Algunos elementos que se toman en cuenta para el reconocimiento y recompensa de las labores de los empleados son: adecuar la recompensa a las personas, adecuar el premio a 
lo logrado y ser oportuno y específico. Para Serrano (2012) el reclutamiento y la selección son procesos claves para aprovisionarse de las cualificaciones y competencias necesarias de lo que demanda la organización, así como la evaluación del capital humano. Para el autor cuando una empresa lleva a cabo una evaluación del rendimiento de sus trabajadores persigue objetivos tanto administrativos como motivacionales, buscando, en última instancia, una mejora de sus resultados organizativos.

Sobre la clasificación de las recompensas estas pueden ser intrínsecas o no monetarias consisten en la satisfacción que una persona recibe del puesto mismo o del entorno del puesto en el que trabaja y engloba aspectos tales como reconocimiento y estatus, buena comunicación, ascensos, mayor autonomía, etc. Las recompensas extrínsecas o monetarias consisten en el pago que recibe una persona en forma de salarios, sueldos, incentivos y prestaciones a cambio de su trabajo. La adecuada amalgama de los tipos de recompensa de acuerdo al comportamiento de los empleados, a las metas empresariales, a las necesidades de los empleados, hará que se logren los objetivos de rentabilidad esperados de las compensaciones diseñadas.

Para las teorías de la motivación, la compensación financiera es una recompensa valiosa y puede motivar a los trabajadores a desempeñarse mejor, puede satisfacer diversas necesidades y reforzar los comportamientos deseados (Gordon, 1997). Sin embargo, dada la complejidad y la dificultad de la aplicación correcta de la compensación financiera, las organizaciones con frecuencia la usan mal como recompensa. Por esto, las organizaciones podrían no satisfacer las necesidades que pretenden llenar con la compensación financiera.

Según García (2006) las compensaciones influyen en las actitudes y comportamientos de los empleados en el trabajo, estimulándolos a ser más productivos. 
Todos los gerentes buscan un mejor desempeño, una productividad más elevada y una menor rotación del personal, lo cual explica la atención seria que la compensación recibe de la alta dirección. Bajo este punto de vista el autor recomienda que, en el sistema de compensaciones de una empresa, las organizaciones luchen por alcanzar la equidad, que es la percepción de los trabajadores a que se les está tratando con justicia. La equidad debe ser justa para todas las partes involucradas y se la debe percibir como tal, situación que de entrada genera dificultad por el carácter singular de los empleados. Por esta razón, la teoría sobre la compensación financiera nunca ha podido proporcionar una respuesta completamente satisfactoria al interrogante de ¿qué es lo que merece una persona por el desempeño de su puesto? La mayoría de los autores señalan como objetivos tradicionales de los sistemas de compensación financiera los de atraer, retener y motivar a los trabajadores en las organizaciones.

Gordon (1997), al definir un sistema de compensación financiera menos tradicional, considera que los sistemas de compensación financiera de una organización incorporan los mecanismos formales para mejorar o reforzar el desempeño de calidad. Para el, las organizaciones tienden a considerar la remuneración como un gasto y a la vez como un activo. Es un gasto en el sentido de que refleja el costo de la mano de obra. Y es un activo cuando persuade a los empleados de desarrollar su mejor esfuerzo y permanecer en su puesto. Los programas de compensación financiera y, en general, de compensaciones influyen en las actitudes comportamientos de los empleados en el trabajo, estimulándolos a ser más productivos. Todos los gerentes buscan un mejor desempeño, una productividad más elevada y una menor rotación del personal, lo cual explica la atención seria que la compensación recibe de la alta dirección. 
Las organizaciones buscan atraer, motivar y retener a los empleados competentes (Wayne Mondy, Sphr, 1997). Puesto que el cumplimiento de estas metas se logra en gran parte por medio del sistema de compensaciones de una empresa, las organizaciones luchan por alcanzar la equidad, para que la percepción de los trabajadores sea que se les está tratando con justicia. La equidad debe ser justa para todas las partes involucradas y se la debe percibir como tal, situación que de entrada genera dificultad por el carácter singular de los empleados.

\section{Conclusión}

En cualquier tipo de organización, el talento humano es un factor importante para alcanzar los objetivos propuestos. Es por esto que claramente debiera existir un sistema metódico, objetivo y eficiente de evaluación del desempeño con el fin de fortalecer sus potencialidades y conocer sus habilidades, destrezas y actitudes para alcanzar resultados. Así mismo esta evaluación es fundamental para los procesos de selección, el desarrollo de ascensos y la asignación de sueldos y salarios.

Torres (2005) considera que la gestión del capital humano es determinante para los resultados de negocio, entonces un mejor despliegue y aplicación apropiada de prácticas de talento humano debería correlacionar con más altos beneficios financieros. Para el autor las empresas normalmente conocen el costo de las personas, pero desconocen el valor que aportan. Esto refuerza la visión de las personas como costos a ser minimizados y no como activos valiosos para maximizar su contribución. Como consecuencia, la gestión de recursos humanos se ha focalizado más en la reducción de costos laborales. Es necesario superar esta visión de las personas como costos para las organizaciones. Comenzar a comprender y medir los impactos en resultados de negocio que tiene el capital humano, 
permitirá asumir un nuevo enfoque de gestión del talento humano como una inversión que genera valor.

\section{Referencias:}

Ahumada F.L. (2002). El Aprendizaje Organizacional Desde una Perspectiva Evolutiva y Constructivista de la Organización. Revista de Psicología. 11(1). P: 139-148. Universidad de Chile Santiago, Chile. Disponible en: http://www.redalyc.org/pdf/264/26411110.pdf

Arenas, M.G. (2002). La contratación de trabajadores a través de empresas de servicios temporales. Precedente. Disponible en: https://www.icesi.edu.co/precedente/ediciones/2002/9ArenasMonsalve.pdf

Díaz, L.E. (2005). Relaciones laborales especiales: las empresas de trabajo temporal y las cooperativas. ¿Qué pueden hacer los sindicatos?. Revista de Ciencias Sociales. 11 (1). P: 131-148. Universidad del Zulia Maracaibo, Venezuela. Disponible en: http://www.redalyc.org/pdf/280/28011109.pdf

Fernández H.E. (2004). Causas de la utilización del empleo temporal y la subcontratación: Análisis empírico de las industrias extractivas en León Estudios de Economía Aplicada. 22 (2). P: 1-30. Asociación Internacional de Economía Aplicada Valladolid, España. Disponible en: http://www.redalyc.org/pdf/301/30122212.pdf

García, C.O. (2006). La compensación financiera: una medida del valor del trabajador Pensamiento \& Gestión. 21, p. 182-233. Universidad del Norte Barranquilla, Colombia. Disponible en: http://www.redalyc.org/pdf/646/64602107.pdf

García, S.M. (2009). Los macro-procesos: un nuevo enfoque en el estudio de la Gestión Humana. Pensamiento \& Gestión. 27. P: 162-200. Universidad del Norte 
Barranquilla, Colombia. Disponible en:

http://www.redalyc.org/pdf/646/64612782006.pdf

Prieto, A. \& Martínez, M. (2004). Sistemas de información en las organizaciones: Una alternativa para mejorar la productividad gerencial en las pequeñas y medianas empresas. Revista de Ciencias Sociales. 10 (2) P: 322-337. Universidad del Zulia Maracaibo, Venezuela. Disponible en: http://www.redalyc.org/pdf/280/28010209.pdf

Rodríguez, T.E. (2010). Guía para establecer empleos temporales en las empresas sociales del estado ESE. Gobierno de Colombia, Prosperidad para todos. Disponible en:http://www.minsalud.gov.co/Documentos\%20y\%20Publicaciones/Gu\%C3\%AD a\%20para\%20crear\%20Empleos\%20Temporales\%20en\%201as\%20Empresas\%20S ociales\%20del\%20Estado\%20ESEs.pdf

Schwartzman K.R. (2000). Capacitación Basada en Normas de competencia laboral. Una alternativa para el nuevo milenio en cuestión de capacitación Revista del Centro de Investigación. Universidad La Salle, 4(14). P: 87-93 Universidad La Salle Distrito Federal, México. http://www.redalyc.org/pdf/342/34201411.pdf

Serrano, S.J. \& Barba, A.M. (2012). La gestión de recursos humanos en las corporaciones locales. Cuadernos de Gestión. 12 (2). P: 149-168. Universidad del País Vasco. Euskal Herriko Unibertsitatea, Vizcaya, España. Disponible en: http://www.redalyc.org/pdf/2743/274324369007.pdf

Trigueros, P.S.; Pérez: G.D. \& Solana, G.P: (2014). Tecnologías de la información y generación de valor en el negocio: Un análisis en pymes industriales Intangible 
Capital. 10 (3), p. 639-663. Universidad Politécnica de Catalunya Barcelona, España. Disponible en: http://www.redalyc.org/pdf/549/54932488011.pdf

Torres, O.J. (2005). Enfoques para la medición del impacto de la Gestión del Capital Humano en los resultados de negocio. Pensamiento \& Gestión. 18. P: 151-176 Universidad del Norte Barranquilla, Colombia. Disponible en: http://www.redalyc.org/pdf/646/64601807.pdf 